\title{
Study on Algorithm of Multidimensional Sets Sequential Patterns Mining Based on Identification of Position
}

\author{
Yan Liü, a , Xiaowei Qin ${ }^{1, b}$ and Yan Zou ${ }^{1, c}$ \\ ${ }^{1}$ College of Computer and Information Engineering, Chifeng University, China \\ acfxyly5962@163.com, 'pxw3409@163.com, czouyankb@163.com
}

Keywords: multidimensional sets; support; sequential patterns mining

\begin{abstract}
Introduce the concepts related to multidimensional set sequence database, and in order to mine a special multidimensional set sequence database, in which all the sequences have the same length, items inside each dimension are ordered, and dimensions are unordered, we propose an algorithm of multidimensional sets sequential patterns mining. This algorithm is on the basis of the multidimensional sets sequential patterns mining based on bitmap, first uses the representation of multidimensional sets sequential database based on identification of position to mine the single frequent itemsets, and then uses the bitmap representation to mine the multidimensional frequent sequences. In each mining, the algorithm only records frequent itemsets of single dimensional element and corresponding frequent sequences, which can reduce memory overhead, improve the counting efficiency of support and mining speed.
\end{abstract}

\section{Introduction}

Data mining has been widely used in various fields, and the mined data patterns have been extended from single data sets insde dimesions to multiple data sets among dimensions, it is so called multidimensioanl mining ${ }^{[1][2]}$. The system construction of college students' innovation ability training and the research on related factor indicate that ${ }^{[3]}$ the cultivation of creativity is related with many factors, such as education system, education process, family environment, personal hobbies, enterprise culture, government advocacy and social environment. Each element contains several attributes, such as education process includes the accumulation of knowledge, the cultivation of creative thinking, innovation practice, innovation achievement display etc. Each attribute contains some different items, such as innovation practice includes experimental innovation activities, innovation activities of science and technology, social practice, innovation practice base etc. Each item also has different values. These elements compose a multidimensional transaction database. How to mine the elements closely related to college students' innovation ability development from this database, and to use in the education process of students' innovation ability in order to improve the students' innovation ability, is not only related to personal development of students, but also give an important scientific support to the cultivation of application and creative ability of students. Based on this requirement, we have studied on multidimensional sets of sequential patterns mining.

\section{Concepts Related to Multidimensional Sets ${ }^{[4][5]}$}

Single dimensional set: The set of items denoted by $I=\left\{i_{1}, i_{2}, \ldots, i_{m}\right\}(m \geq 0)$, in which $i_{\mathrm{j}}(1 \leq j$ $\leq m$ ) is called item, $I$ sreprents single dimensional set on attribute set $E$ whose value domain is $V$, and $i_{\mathrm{j}} \in V$. When $m=0, I$ is empty set; When $m=1, I$ is called the single dimensional set with single item; When $m \geq 1, I$ is called real single dimensional set.

The length of single dimensional set: The number of items included in single dimensional set.

Multidimensional set: Suppose $A=\left\{E_{1}, \ldots, E_{\text {dim }}\right\}(\operatorname{dim} \geq 1)$ is any attribute set, is the single dimensional set on any attribute set, $\operatorname{dim}$ is the number of dimension, the value domain of each attribute is $V_{1}, \ldots, V_{\text {dim }}$ respectively, the multidimensional set $t=\left\{s_{1}, \ldots, s_{d i m}\right\}(\operatorname{dim} \geq 1)$, in which $s_{i}(1 \leq i \leq \operatorname{dim})$ is the single dimensional set on attribute $E_{\text {dim }}$, i.e. $s_{i} \subseteq V_{i}$, then $t$ is called 
multidimensional set or dim-dimensional set on attribute set $A$. If $s_{i}$ is composed of the single dimensional set with single item, then we call it multidimensional set with single item, else we call it real multidimensional set. The number of single dimensioanl sets contained in multidimensional set is called the length of multidimensional set.

Contain: Suppose $t$ and $s$ are two multidimensional sets on attribute set $A$, for any attribute $E_{\text {dim }}$ $\in A$ (dim $\geq 1), t_{i}$ is the multidimesional set of $t$ on attribute $E_{\text {dim }}, s_{i}$ is the multidimesional set of $s$ on attribute $E_{\text {dim }}$, the clusion relation $s_{i} \subseteq t_{i}$ are both ture. So we call multidimensional set $t$ contains multidimensional $s$ denoted by $t \geq s$.

Multidimensional sets sequential patterns: Suppose $D$ is a dim-dimensional set on $A=\left\{E_{1}, \ldots\right.$, $\left.E_{\text {dim }}\right\}(\operatorname{dim} \geq 1), B$ and $C$ are attribute sets on $A, B \subset A$ and $C \subset A$ and $B \cap C=\varnothing$. Suppose $r$ is a real multidimensional set on attribute set $B, t$ is a real multidimensional set on attribute set $C$. Given a support threshold $\xi$, if the number of multidimensional set $s$ that contains both $r$ and $t$ in $D$ is larger than $\xi$, then call $\mathrm{r} \Rightarrow \mathrm{t}$ is multidimensional sets association rules based on attribute relations $B$ and $C$.

\section{Multidimensional Sets Database}

According to the above definition of multidimensional sets, we give the multidimensional sets databse to be mined as shown in Table 1. Each itemset represents a single dimensional set on each attribute, each sequence represents multidimensional set on attribute sets. To meet the need of the analysis of elements related to the innovation ability of college students, we call the attributes in this database elements $E_{\text {dim }}$ that represent the elements related to the innovation ability of college students. Each sequence with the same dimension number dim is composed of single dimensional sets. Items are ordered inside each dimension, but not ordered among dimesions. Items in different dimensions represent different meaning, this is different from definiton of traditional transaction database.

Transform the database in Table 1 into the form in Table 2, there are 3 sequences that represent 3 multidimensional sets, the length of each sequence is 3 that represents three itemsets, each itemset represents one single dimensional set on element $E_{\text {dim }}$. In each multidimensional set, the order of itemsets can not be changed, e.g. ( $b c a$ ) and ( $a b c$ ) are two different itemsets in this paper and represent different meaning. The item $a$ of the single dimensional set on $E_{1}$ is different from the item $a$ in $E_{2}$ and $E_{3}$, this is just to simplify the representation and no effect on mining result.

Table 1 Multidimensional sets database

\begin{tabular}{|c|c|}
\hline Sid & Sequence \\
\hline 1 & $<(b c a)(e a)(b a c)>$ \\
\hline 2 & $<(a b d)(e c a)(a b)>$ \\
\hline 3 & $<(b c d a)(d e b)(a c b)>$ \\
\hline
\end{tabular}

Table 2 Multidimensional sets sequence database

\begin{tabular}{|c|c|c|c|}
\hline \multirow{2}{*}{ Sid } & \multicolumn{3}{|c|}{ Itemsets of Sequence on $\boldsymbol{E}_{\operatorname{dim}}$} \\
\cline { 2 - 4 } & $\boldsymbol{d i m}=\mathbf{1}$ & $\boldsymbol{d i m}=\mathbf{2}$ & $\boldsymbol{\operatorname { d i m }}=\mathbf{3}$ \\
\hline 1 & $(b c a)$ & $(e a)$ & $(b a c)$ \\
\hline 2 & $(a b d)$ & $(e c a)$ & $(a b)$ \\
\hline 3 & $(b c d a)$ & $(d e b)$ & $(a c b)$ \\
\hline
\end{tabular}

\section{The Algorithm of Multidimensional Sets Sequential Patterns Mining Based on Identification of Position}

The idea of the algorithm. On the basis of existing sequential patterns mining algorithm ${ }^{[6]}$, to meet the need of new data model, we propose an algorithm of multidimensional sequential patterns mining based on positional identification. This algorithm uses database based on identification of position to mine frequent itemsets that can identify the order among items accurately, and uses bitmap representation to mine frequent sequences that improves counting efficiency. In each mining, the algorithm only records frequent itemsets of one dimensional element and corresponding association rules, which can reduce memory overhead. The idea of the algorithm is described as follows:

(1) Generate the sequence database based on the identification of position Pos_D.

(2) On element $E_{\text {dim }}$, mine the frequent itmesets $F I_{k_{-} \text {dim }}$ first, and then mine the corresponding frequent sequence $F S_{\text {dim }}$. 
(3) Mine frequent itemsets $F I_{k_{-} \text {dim }}$. Generate candidate itemsets $C I_{k_{-} \text {dim }}$ through sequential patterns tree, and then count the support using database based on position identification to generate the frequent sequence $F I_{k_{d} \text { dim }}$ on $E_{\text {dim }}$, and record the frequent itemsets in table Pos_FI $I_{\text {dim }}$.

(4) Mine frequent sequence $F S_{\text {dim }}$. Generate candidate sequences $C S_{\text {dim }}$ through sequential patterns tree constructed by the frequent sequence $F S_{d i m-1}$ of the dimension before current dimension element and frequent itemsets $F I_{k_{-} \text {dim }}$ of current dimension element, and count the support by bitmap representation to generate the multidimensional frequent sequence $F S_{\text {dim }}$, and then record the frequent sequences in table Bit_FS $S_{\text {dim }}$.

Description of algorithm. Input: Multidemisional sets sequence dababase based on identification of position Pos_D, min_support

Output: Multidemisional sets frequent sequential pattern $F S_{\text {dim }}$

(1) for each dimension element $E_{\text {dim }}$

(2) $F I_{1 \_ \text {dim }}=$ generate $\left(P o s \_D\right) ; / / F I_{1 \_ \text {dim }}$ is frequent 1 -itemsets on the dimth dimesion element $E_{\text {dim }}$

(3) for $\left(k=2 ; F I_{k-1 \_d i m} \neq \varnothing ; k++\right) / /$ generate frequent $k$-itemsets $F I_{k_{-} \operatorname{dim}}$ on $E_{\text {dim }}$

(4) $\quad C I_{k \_d i m}=$ generate_CI $\left(F I_{k-1 \_d i m}\right)$; // $C I_{k \_d i m}$ is candidate $k$-itemsets on $E_{\text {dim }}$

(5) $F I_{k \_d i m}=$ generate_frequent_kitemsets $\left(C I_{k \_d i m}, P o s_{-} F I_{d i m}\right)$; //Pos_FI $I_{\text {dim }}$ is frequent table on $E_{\text {dim }}$

(6) if $(\operatorname{dim}==1) / /$ generate frequent 1-sequences $F S_{1}$

(7) $F S_{1}=F I_{k_{-} \text {dim }} ; / / F S_{1}$ is composed of frequent $k$-itemsets on the 1 st demision element $E_{1}$

(8) else if ( $F S_{d i m-1} \neq \varnothing \& \& F I_{k_{-} d i m} \neq \varnothing$ )//generate multidemisioanl frequent sequential patterns $F S_{\text {dim }}$

(9) $C S_{\text {dim }}=$ generate_CS $\left(F S_{\text {dim-1 }}, F I_{k_{-} \text {dim }}\right) ; \quad / / C S_{\text {dim }}$ is the candidate dim-sequences

(10) $F S_{\text {dim }}=$ generate_frequent_sequences $\left(C S_{\text {dim }}, P o s \_F I_{\text {dim }}, B i t \_F S_{\text {dim }}\right)$;

(11) return $F S_{\text {dim }} ; / / F S_{\text {dim }}$ is frequent dim-sequences

\section{The Description of Algorithm Flow}

This paper takes multidimensional sets sequence database in Table 2 as example to describe the algorithm flow, and supposes the minimum support count is min_support $=2$.

Generate multidimensional sequence database based on identification of position Pos_D. Save the sequence database of Table 2 into Pos_D shown in Table 3. Record the occurrence position of each item on each dimension element in Pos_D. For example, the 1 st element $E_{1}$ of sequence 1 is the itemset ( $b c a$ ), in which the occurrence position of item $a$ is 3 , so noted as $\operatorname{Pos}(a)=3$, the occurrence position of item $b$ is 1 , so noted as $\operatorname{Pos}(b)=1$, the occurrence position of item $c$ is 2 , so noted as $\operatorname{Pos}(c)$ $=2$, while item $d$ doesn't occur, so noted as $\operatorname{Pos}(d)=0$.

\section{Generate frequent $\boldsymbol{k}$-itemsets $\boldsymbol{F} \boldsymbol{I}_{\boldsymbol{k}_{-} \text {dim }}$ on each dimension element $\boldsymbol{E}_{\text {dim }}$.}

Generate frequent $k$-itemsets $F I_{k_{-1}}$ on the 1 st dimension element $E_{1}$.

Generate frequent 1-itemsets $\boldsymbol{F I}_{\mathbf{1}_{-1}}$ on $\boldsymbol{E}_{\mathbf{1}}$. (1) Take $\operatorname{dim}=1$, Sid $=1$, scan the item $a$ in database Pos_D longitudinally. If the value of $\operatorname{Pos}(a)$ is not 0, add 1 to support(a). (2) Add 1 to Sid, continue to perform the above operation until Sid $=3$. If $\operatorname{support}(a) \geq \min \_$support, then $(a) \in F I_{1 \_1}$, and record in Pos_FI dim as shown in Table 4; else $(a) \notin F I_{1 \_1}$. (3) Continue to scan next item $b$ unitl to $d$, all of the frequent 1 -itemsets $F I_{1-1}$ on 1st dimension element $E_{1}$ are generated, which are $(a)$, $(b),(c),(d)$ as shown in Table 4.

Table 3 Multidimensional sets sequence database based on identification of position Pos_D

\begin{tabular}{|c|c|c|c|c|c|c|c|c|c|c|c|c|}
\hline \multirow{3}{*}{ Sid } & \multicolumn{12}{|c|}{$\operatorname{Pos}\left(E_{\operatorname{dim}}\right)$} \\
\hline & \multicolumn{4}{|c|}{$\operatorname{dim}=1$} & \multicolumn{4}{|c|}{$\operatorname{dim}=2$} & \multicolumn{4}{|c|}{$\operatorname{dim}=3$} \\
\hline & $a$ & $b$ & $c$ & $d$ & $a$ & $\boldsymbol{b}$ & $C$ & $d$ & $e$ & $a$ & $b$ & C \\
\hline 1 & 3 & 1 & 2 & 0 & 2 & 0 & 0 & 0 & 1 & 2 & 1 & 3 \\
\hline 2 & 1 & 2 & 0 & 3 & 3 & 0 & 2 & 0 & 1 & 1 & 2 & 0 \\
\hline 3 & 4 & 1 & 2 & 3 & 0 & 3 & 0 & 1 & 2 & 1 & 3 & 2 \\
\hline
\end{tabular}


Generate candidate 2-itemsets $\boldsymbol{C I}_{2 \_1}$ on $\boldsymbol{E}_{1}$. Construct the sequential patterns tree ${ }^{[8]}$, root is the root node. Its subnodes formed the first layer nodeds are the first items of $C I_{2}{ }_{1}$ composed of all the frequent 1-itemsets on 1st dimension element. The subnodes of first layer nodes composed of their sibling nodes, formed the second layer nodes, and are the second items of $C_{2_{2}}$. For example, in Fig. 1, the father node of $(a)$ is root, the subnodes of root are $(a),(b),(c),(d)$, so the subnodes of $(a)$ are its sibling nodes $(b),(c),(d)$. And the candidate 2-itemsets $C I_{2 \_}$are $(a b),(a c),(a d)$, $(b a),(b c),(b d),(c a),(c b),(c d),(d a),(d b),(d c)$.

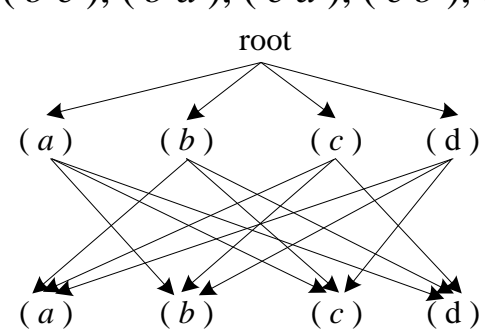

Fig.1 Candidate 2_itemsets $C I_{2 \_1}$ on $E_{1}$

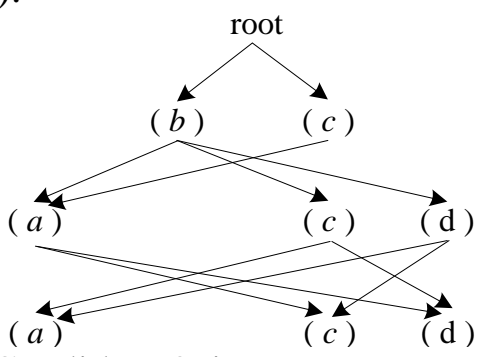

Fig.2 Candidate 3_itemsets $C I_{3 \_1}$ on $E_{1}$

Generate frequent 2-itemsets $\boldsymbol{F I}_{2_{-} \mathbf{1}}$ on $\boldsymbol{E}_{\mathbf{1}}$. (1) Take one candidate 2-itemset $C_{2_{2} 1}$, such as ( $b a$ ). Take $\operatorname{dim}=1$, $\operatorname{Sid}=1$, scan $(b)$ and $(a)$ of $F I_{1-1}$ in Table 4. If $\operatorname{Pos}(a) \neq 0, \operatorname{Pos}(b) \neq 0, \operatorname{Pos}(a)>$ $\operatorname{Pos}(b)$ are all true, which indicate that $(a)$ and $(b)$ both occur, and $(a)$ is located behind $(b)$, so add 1 to support $(a b)$, and record $\operatorname{Pos}(b a)=\operatorname{Pos}(a)$; else record $\operatorname{Pos}(b a)=0$. (2) Increase Sid until Sid = 3. If support $(a b) \geq m i n \_s u p p o r t$, then ( $\left.b a\right) \in F I_{2 \_1}$, record ( $a b$ ) in table Pos_FI dim, as shown in Table 4; else ( $b a$ ) $\notin F I_{2 \_}$. (3) Continue to take next candidate 2-itemset $C I_{2 \_1}$ until to the end, and then generate all the frequent 2-itemsets $F I_{2_{-1}}$ on $E_{1}$, which are ( $\left.b a\right),(b c),(b d)$ and $(c a)$.

Table 4 Frequent itemsets table on the dimth element $E_{\text {dim }}-P o s \_F I_{\text {dim }}$

\begin{tabular}{|c|c|c|c|c|c|c|c|c|c|c|c|}
\hline \multirow{3}{*}{ Sid } & \multicolumn{11}{|c|}{$F I_{k-\operatorname{dim}}(\operatorname{dim}=1)$} \\
\hline & \multicolumn{5}{|c|}{$F I_{1-1}(k=1)$} & \multicolumn{5}{|c|}{$F I_{2-1}(k=2)$} & \multirow{2}{*}{$\begin{array}{c}F I_{3-1}(k=3) \\
(\text { bca })\end{array}$} \\
\hline & (a) & $(b)$ & & & $(d)$ & \multicolumn{2}{|c|}{$(b a)$} & $(b c)$ & $(b d)$ & $(c a)$ & \\
\hline 1 & 3 & 1 & & & 0 & 3 & \multicolumn{2}{|c|}{2} & 0 & 3 & 3 \\
\hline 2 & 1 & 2 & & & 3 & 0 & \multicolumn{2}{|c|}{0} & 3 & 0 & 0 \\
\hline 3 & 4 & 1 & & & 3 & 4 & \multicolumn{2}{|c|}{2} & 3 & 4 & 4 \\
\hline \multirow{3}{*}{ Sid } & \multicolumn{6}{|c|}{$F I_{k_{-} \operatorname{dim}}(\operatorname{dim}=2)$} & \multicolumn{5}{|c|}{$F I_{k_{-} \operatorname{dim}}(\operatorname{dim}=3)$} \\
\hline & \multicolumn{3}{|c|}{\begin{tabular}{c|c}
$F I_{1-2}(k=1)$ \\
$(a)$
\end{tabular}} & \multirow{2}{*}{\multicolumn{3}{|c|}{$\begin{array}{c}F I_{2 \_2}(k=2) \\
(e a)\end{array}$}} & \multicolumn{3}{|c|}{$F I_{1 \_3}(k=1)$} & & $F I_{2 \_3}(k=2)$ \\
\hline & (a) & (e & & & & & (a) & $(b)$ & (c) & $(a b$ & $(a c)$ \\
\hline 1 & 2 & 1 & & & 2 & & 2 & 1 & 3 & 0 & 3 \\
\hline 2 & 3 & 1 & & & 3 & & 1 & 2 & 0 & 2 & 0 \\
\hline 3 & 0 & 2 & & & 0 & & 1 & 3 & 2 & 3 & 2 \\
\hline
\end{tabular}

Note: Pos_FI $I_{\text {dim }}$ only records frequent $k$-itemsets on one dimension element at one time, here lists records for three times.

Generate candidate 3-itemsets $\boldsymbol{C I}_{3_{3} \mathbf{1}}$ on $\boldsymbol{E}_{\mathbf{1}}$. In sequential patterns tree, the first layer nodes are the first items of frequent 2-itemsets $F_{2}$, the second layer nodes are the second items of frequent 2-itemsets $\mathrm{FI}_{2}$, , the third layer nodes are composed of the sibling nodes of the second layer nodes. For example, in Fig. 2, the father node of $(a)$ is $(b)$ whose subnodes are $(a),(c)$ and $(d)$, so the subnodes of $(a)$ are its sibling nodes $(c)$ and $(d)$. And the candidate 3-itemsets $C I_{3 \_1}$ are ( $b$ $a c),(b a d),(b c a),(b c d),(b d a),(b d c),(c a d)$.

Generate frequent 3-itemsets $\boldsymbol{F I}_{3 \_}$on $\boldsymbol{E}_{1}$. (1) Take one candidate 3-itemset $C_{3}$ _1 , e.g. ( $b c$ $a)$. Take $\operatorname{dim}=1, \operatorname{Sid}=1$, scan $(b c)$ and $(a)$ in Table 4. If $\operatorname{Pos}(b c) \neq 0, \operatorname{Pos}(a) \neq 0, \operatorname{Pos}(a)>\operatorname{Pos}(b c)$ are all true, which indicate $(a)$ and $(b c)$ both occur, and $(a)$ is located behind $(b c)$, then add 1 to support $(b c a)$, and record $\operatorname{Pos}(b c a)=\operatorname{Pos}(a)$; else record $\operatorname{Pos}(b c a)=0$. (2) Increase Sid until Sid = 3. If $\operatorname{support}(b c a) \geq$ min_support, then ( $b c c a) \in F I_{3 \_}$, record ( $b c c a$ ) in table Pos_FI dim as shown in Table 4; else ( $b$ c $a$ ) $\notin F I_{3-1}$. (3) Continue to take next candidate 3-itemset $C_{3_{3} 1}$ until to the end, then generate all the frequent 3-itemsets $C I_{3-1}$ on $E_{1}$, which is ( $b$ c $a$ ).

Generate candidate $\boldsymbol{k}$-itemsets $\boldsymbol{C I}_{\boldsymbol{k}_{-1}}$ on $\boldsymbol{E}_{1}$. Construct sequential patterns tree, the $(k-1)$ th items of frequent $(k-1)$-itemsets $F I_{k-1 \_1}$ compose the $(k-1)$ th layer nodes, whose sibling nodes compose the subnodes of it. Use generate_C $\left(F I_{k-1 \_d i m}\right)$ to generate candidate $k$-itemsets $C I_{k_{-}}$. 
Generate frequent k-itemsets $\boldsymbol{F I}_{\boldsymbol{k}_{-1}}$ on $\boldsymbol{E}_{\mathbf{1}}$. (1) Take one candidate $k$-itemset $C I_{k_{-}}$, take dim $=1$, Sid $=1$, scan the top $k-1$ item and $k$ th item of $C I_{k_{-}}$in Table 4 . If both values are not 0 , and the value of $k$ th item is greater than top $k-1$ item, then add 1 to $\operatorname{support}\left(C I_{k_{-}}\right)$, and record the value of $\operatorname{Pos}\left(C I_{k_{-1}}\right)$ the same as the value of $k$ th item; else record the value of $\operatorname{Pos}\left(C I_{k_{-1}}\right)$ as 0 . (2) Increase Sid until Sid $=3$. If $\operatorname{support}\left(C I_{k_{-1}}\right) \geq \min \_$support, then $C I_{k_{-1}} \in F I_{k_{-1}}$, record $C I_{k_{-1}}$ in table Pos_FI dim, as shown in table 4 ; else $C I_{k_{-1}} \notin F I_{k_{-}}$. (3) Continue to take next candidate $k$-itemset $C I_{k_{-}}$, until to the end, then generate all the frequent $k$-itemsets $F I_{k_{-1}}$ on $E_{1}$.

Generate frequent $\boldsymbol{k}$-itemsets $\boldsymbol{F I}_{\boldsymbol{k}_{-} \text {dim }}$ on $\boldsymbol{E}_{\text {dim }}$. Add 1 to dim, the process of algorithm is the same as section 2.1, until to the last dimension element. Record the frequent $k$-itemsets $F I_{k \_d i m}$ on the dimth dimension element in table $F I_{k \_ \text {dim }}$.

Generate multidimensional frequent sequences $F S_{\text {dim }}$.

Generate frequent 1-sequences $\boldsymbol{F} \boldsymbol{S}_{\mathbf{1}}$. If $\operatorname{dim}=1$, frequent 1-sequences $F S_{1}$ is composed of the frequent $k$-itemsets $F I_{k_{-1}}$ on $E_{1}$, then save $F I_{k_{-1}}$ of Table 4 into frequent sequence table Bit_FS $S_{\text {dim }}$ in the form of bitmap, as shown in Table 5 . The value is not 0 in Table 4 will be recorded as 1 in Table 5 which represents occurrence, and the value is 0 will be still recorded as 0 which represents not occurrence. For example, in Table 4, if $\operatorname{Sid}=1, \operatorname{Pos}(a)=3$, then record $\operatorname{Bit}(a)=1$ in Table 5. And then , clear table Pos_FI $I_{\text {dim }}$ in order to record the frequent $k$-itemsets on next dimension element.

Generate candidate 2-sequences $\boldsymbol{C S}_{2}$. If $\mathrm{dim}=2$, construct sequential patterns tree, the root node is root. Its subnodes formed the first layer nodes are the first itemsets of $C S_{2}$ composed of all the frequent 1-sequences $F S_{1}$. The subnodes of first layer nodes formed the second layer nodes are the second itemsets of $C S_{2}$, which are composed of all the frequent $k$-itemsets $F I_{k \_2}$ on the 2nd dimension element $E_{2}$ in Table 4. As shown in Fig. 3.

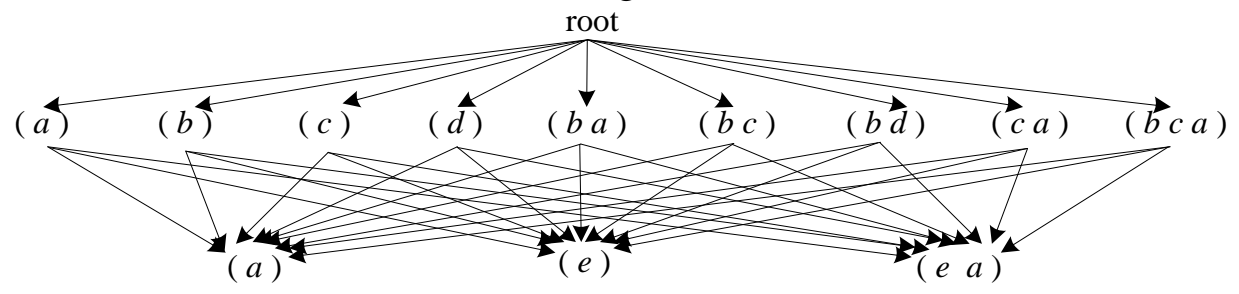

Fig.3 Candidate 2_sequence $C S_{2}$

Generate frequent 2-sequence $\boldsymbol{F S}_{\mathbf{2}}$. (1) Record $F S_{1}$ of table Bit_FS $S_{\text {dim }}$ into table Temp_FS $S_{\text {dim }}$, as shown in Table 6. (2) Take one candidate 2-sequence $C S_{2}$, e.g. ( c a ) ( e ). Take Sid=1, scan ( c $a$ ) in Table 6, and scan ( $e$ ) with $\operatorname{dim}=2$ in Table 4. If both Bit $(c a)$ and $\operatorname{Pos}(a)$ are not 0, which indicates ( $c$ a ) and ( $e$ ) all occur, then add 1 to support $((c a)(e))$, and record $\operatorname{Bit}((c a)(e))=1$; else record Bit $((c a)(e))=0$. (3) Increase Sid until Sid = 3. If support $((c a)(e)) \geq$ min_support, then $(c a)(e)$ $\in F S_{2}$, record ( $c a$ ) ( $e$ ) in table Bit_FS $S_{\text {dim }}$, as shown in Table5; else ( c $a$ ) ( e $) \notin F S_{2}$. (4) Continue to take next candidate 2-sequence $C S_{2}$, until to the end, and generate all the frequent 2-sequences $F S_{2}$, they are $(a)(a),(a)(e),(a)(a e),(b)(a),(b)(e),(b)(a e),(c)(e),(d)(e)$, $(b a)(e),(b c)(e),(b d)(e), \quad(c a)(e),(b c a)(e)$. As shown in Table5.

And then, clear table Pos_FI $I_{\text {dim }}$ to record the frequent $k$-itemsets on next dimension element.

Table 5 Frequent sequences table Bit_FS $S_{\text {dim }}$

\begin{tabular}{|c|c|c|c|c|c|c|c|c|c|c|c|c|c|c|c|}
\hline \multirow{3}{*}{ Sid } & \multicolumn{15}{|c|}{ Bit $\left(F S_{\text {dim }}\right)$} \\
\hline & \multicolumn{10}{|c|}{$F S_{1}(\operatorname{dim}=1)$} & \multicolumn{5}{|c|}{$F S_{2}(\operatorname{dim}=2)$} \\
\hline & (a) & (b) & (c) & $(d)$ & $(b a)$ & $(b c)$ & $(b d)$ & $(c a)$ & \multicolumn{2}{|c|}{$(b c a)$} & \multicolumn{2}{|c|}{$(a)(a)$} & \multicolumn{2}{|c|}{$(a)(e)$} & $(a)(a e)$ \\
\hline 1 & 1 & 1 & 1 & 0 & 1 & 1 & 0 & 1 & \multicolumn{2}{|c|}{1} & \multicolumn{2}{|c|}{1} & & 1 & 1 \\
\hline 2 & 1 & 1 & 0 & 1 & 0 & 0 & 1 & 0 & \multicolumn{2}{|c|}{0} & \multicolumn{2}{|c|}{1} & & 1 & 1 \\
\hline 3 & 1 & 1 & 1 & 1 & 1 & 1 & 1 & 1 & \multicolumn{2}{|c|}{1} & \multicolumn{2}{|c|}{0} & & 0 & 0 \\
\hline \multirow{3}{*}{ Sid } & \multicolumn{15}{|c|}{ Bit $\left(F S_{\text {dim }}\right)$} \\
\hline & \multicolumn{15}{|c|}{$F S_{2}(\operatorname{dim}=2)$} \\
\hline & \multicolumn{2}{|c|}{$(b)(a)$} & $(b)(e)$ & \multicolumn{2}{|c|}{$(b)(a e)$} & $(c)(e)$ & $(d)(e$ & $(b a)$ & & \multicolumn{2}{|c|}{$(b c)(e)$} & \multicolumn{2}{|c|}{$(b d)(e)$} & $(c a)(e)$ & $\begin{array}{c}\left(\begin{array}{c}b c a \\
(e)\end{array}\right) \\
(e)\end{array}$ \\
\hline 1 & 1 & & 1 & & 1 & 1 & 0 & 1 & & & & 0 & & 1 & 1 \\
\hline 2 & 1 & & 1 & & 1 & 0 & 1 & 0 & & & & 1 & & 0 & 0 \\
\hline 3 & 0 & & 1 & & 0 & 1 & 1 & 1 & & 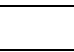 & & 1 & & 1 & 1 \\
\hline
\end{tabular}


Note: Table Bit_FS $S_{\text {dim }}$ only record frequent dim-sequences corresponding to current dimension, here lists two records.

Generate candidate sequence $\boldsymbol{C} \boldsymbol{S}_{\text {dim }}$. Add 1 to $\mathrm{dim}$, construct sequential patterns tree, the root node is root, the first layer nodes are composed of all frequent (dim-1)-sequences $F S_{d i m-1}$, the second layer nodes are composed of all frequent $k$-itemsets on dimth dimension element, and use generate_CS $\left(F S_{k_{-} \text {dim-1 }}, F I_{k \_d i m}\right)$ to generate candidate sequence $C S_{\text {dim }}$.

Generate frequent sequence $\boldsymbol{F S}_{\text {dim }}$. (1) Record $F S_{\text {dim-1 }}$ of table Bit_FS $S_{\text {dim }}$ into table Temp_FS $S_{\text {dim }}$. (2) Take one candidate sequence $C S_{\text {dim }}$. Let $\operatorname{Sid}=1$, scan the top dim-1 itemset of $C S_{\text {dim }}$ in table Temp_FS $S_{\text {dim }}$, and scan the dimth itemset of $C S_{\text {dim }}$ in table Pos_FI $I_{\text {dim }}$, if both are not 0, indicates that both occur, then add 1 to support $\left(C S_{d i m}\right)$, and record $\operatorname{Bit}\left(C S_{\operatorname{dim}}\right)=1$; else record $\operatorname{Bit}\left(C S_{\text {dim }}\right)=0$. (3) Increase $\operatorname{Bit}\left(C S_{\text {dim }}\right)=0$ until to the end. If $\operatorname{support}\left(C S_{\text {dim }}\right) \geq$ min_support, then $C S_{\text {dim }} \in F S_{\text {dim }}$, and record $C S_{\text {dim }}$ in table Bit_FS dim ; else $C S_{\text {dim }} \notin F S_{2}$. (4) Continue to take next candidate sequence $C S_{\text {dim }}$ until to the end, and then generate all the frequent sequenes $F S_{\text {dim }}$.

Table 6 Temporary frequent sequences table Temp_FS $S_{\text {dim }}$

\begin{tabular}{|c||c|c|c|c||c|c|c|c||c||}
\hline \multicolumn{1}{|c||}{ Sid } & \multicolumn{10}{|c||}{$\boldsymbol{F \boldsymbol { S } _ { \mathbf { 1 } }}$} \\
\cline { 2 - 10 } & $(\boldsymbol{a})$ & $(\boldsymbol{b})$ & $(\boldsymbol{c})$ & $(\boldsymbol{d})$ & $(\boldsymbol{b} \boldsymbol{a})$ & $(\boldsymbol{b} \boldsymbol{c})$ & $(\boldsymbol{b} \boldsymbol{d})$ & $(\boldsymbol{c} a)$ & $(\boldsymbol{b} \boldsymbol{c} \boldsymbol{a})$ \\
\hline 1 & 1 & 1 & 1 & 0 & 1 & 1 & 0 & 1 & 1 \\
\hline 2 & 1 & 1 & 0 & 1 & 0 & 0 & 1 & 0 & 0 \\
\hline 3 & 1 & 1 & 1 & 1 & 1 & 1 & 1 & 1 & 1 \\
\hline
\end{tabular}

\section{Conclusions}

The algorithm of multidimensional set sequential patterns mining can mine the sequence database of teaching effect, in which the items are in order while itemsets are not. Through the mining, we can find the relationships among elements. And in the practical application, according to the learning habits of student, as well as the relationship between the grades of courses related to practice teaching and innovation achievements, we can mine the learning state in which they can get good effect of cultivation of innovation ability. This can help to adjust teaching method, fully develop the students' potential, and improve teaching quality. The algorithm can be further optimized in the candidate set and candidate sequence, i.e. effective pruning in the generation of $C I_{k_{-} d i m}$ and $C S_{\text {dim }}$ to improve the efficiency, and should add different weighs in items, which is a study emphasis in further research.

\section{Acknowledgements}

Project supported by the Inner Mongolia Autonomous Region Natural Science Foundation (2011MS0914).

\section{References}

[1] R. Agrawal,R. Srikant. Mining Sequential Patterns. Proc. 11st Int. Conf. Data Engineering. (TaiPei, 1995), pp. 3-14

[2] R.Agrawal, R.Srikant. Mining sequential pattern: Generalizations and performance improvements. Proc. 5th Int. Conf. Extending Database Technology. Vol. 1057(1996), pp.3-17

[3] Li Cunjin. The Realization Way and Mechanism of Training Students' Creative Thinking Ability. Innovation and Entrepreneur Education, Vol.4(2013) , pp.1-5

[4] Zhong Yong, Qi n Xiaoli n, Bao Lei. An Association rule mining Algorithmof multidimensional sets. Journal of Computer Research and development, Vol.43(2006), pp. 2117-2123

[5] Song Shijie, Hu Haping. A Sequential Pattern Mining Algorithm Based on Large Itemset Reuse. Journal Computer Research Development, Vol. 43(2006), pp.68-74

[6] YanLiu, Xiaowei Qin, YanZou. Application of Multivariable Sequential Patterns Mining in Teaching Effect Evaluation. IEEE Computer Society (2013), pp.369-372 